\title{
Study on the Design of the Digital Control Machine-tool Operating System
}

\author{
Ye Tian \\ Tianjin Polytechnic University, Tianjin 300160, China \\ E-mail: 1983tianye@sina.com
}

\begin{abstract}
The ergonomics is an independent subject developed from the beginning of the 20th century, and it is the frontier applied science which studies the comprehensive performances such as comfort ability, production efficiency and safety under various conditions. In the article, a series of human oriented designs were studied for the software and hardware interfaces of the digital control machine-tool based on the theory of the ergonomics and the human-oriented principle.
\end{abstract}

Keywords: Ergonomics, Human-oriented, User, Digital control machine-tool, Design

The digital control machine-tool is the machine-tool which uses the information with the form of code (program instruction) to control the cutter to automatic machining according to appointed work program, sports speed and track. It can be extensively applied in many industries, and when the machining objects change, only the input program instructions need to be changed. Its machining performance is better than general automatic machine, and it can exactly machine complex profile parts, so it is fitter to machine work pieces with middle and small batches, frequent performance change, high precision requirements and complex forms, and acquire better economic effects. With the development of the digital control technology, the varieties of the machine-tool with the digital control system become more and more, and they include lathe, milling machine, boring lathe, drill press, grinding machine, gear cutting machine and electric spark machine tool, and the machining center and turning center which can change the tool automatically and implement multiple working procedures once.

The operating system design of digital control machine-tool can directly influence users' work efficiency and operating comfort level, because good human-machine interface operates simply and effectively, and its guidance function will make users feel comfort and interesting and enhance the using efficiency.

\section{Foreign and domestic actualities and levels of the digital control machine-tool operating system}

With the development of the manufacturing, the tendency of the middle and small lot manufacture is increasingly strengthened, which induces the large potential of the market development of the digital control machine-tool which integrates many specialties such as electron, numerical control and hydraulic pressure automatization. The form of the digital control machine-tool gradually goes to the integration from original dispersedness, and the functions are more complete, and the color and the human-machine interface could more embody the human-computer-environment, and relative technologies and forms all be developed quickly. For the machine-tool itself, its functions and the characters reflected by the function are the first factors what the designers should consider. The function of the machine-tool means that the machine-tool possesses special purpose and functions which are designed by human demands. The machine-tool is the carrier of the functions, and its financial intention is to realize the functions. The machine-tool design and all measures and methods in the manufacturing process are based on the actual functions of the machine-tool in practice. The intention that users purchase it is to enjoy the advantages that it can bring to them.

As viewed from users, the design should be human-oriented, and the machine-tool designed by the designers must harmonize human physiology and psychology, i.e. accord with users' ergonomic characteristics. In the design process, the designers decide the proper modeling scheme or the evaluation of the modeling scheme through human-machine experiment and the computer simulation of human-machine relationship.

Whether for domestic machine-tool or foreign machine-tool, the modelings of machine tool goes to the integration and uniformity, and embody the advancement of the modern manufacturing technology. The modeling of the machine-tool also reflects the technical level of each manufacturer. Comparing with foreign level, the digital control machine-tool of China is less than foreign machine tool not only for the variety but for the quality, and the modeling also lacks in more researches. The modeling of most Chinese machine-tools is simple and dispersed. Because the Eleven Five-year Development Program extends the demands of Chinese digital control machine-tool, so the modeling design of the digital control machine-tool requires more safe creative performance, novel style and practical purpose. 


\section{Problems in the existing digital control machine-tool operating system}

Through the analysis of the foreign and domestic actualities of the digital control machine-tool operating system, the modeling of most machine-tools is simple and goes to integration. The human-machine scale design is not perfect, and the color is single and dark. Excessive components are exposed and lack in safe and integrated characters.

\section{Theoretical meanings}

The typical process of the machine running can be described that human use their hands and feet as the controller, and the machine runs according to the instructions and reflect its running state on the screen, and human accept the information by their eyes and ears and transmit the information to the brain which controls hands and feet to operate through analyzing and judging, and in this way, the working flow can be complete in turn. In this simple human-machine system, the information communication from machine to human exists between display with eyes and ears, and the information channel from human to machine exists between hands and feet with controller, and the interface which implements the bilateral information communication forms an abstract "human-machine interface".

The total working efficiency of the system is decided by not only the working efficiency of machine and human operating efficiency, but the efficiency of information communication of the human-machine interface. The system with users and machine is an information system, and to realize the system object, human and machine should apply proper and reasonable interactive relationship, and the human-machine interface (including control system, operating system and material processing system) all need to be further designed and improved.

The evaluation of the digital control machine-tool is decided by whether it can fulfill operators' work completed requirement. Therefore, it is imperative to explore human comfort operation in the activity and the optimal space scale in the machine-tool system, i.e. study the ergonomic factors in the digital control machine-tool system.

\section{Applied meanings}

Through studying the position and implementation of the control platform, the perceptual knowledge of the human-machine relative relationship can be acquired, which could offer powerful references for the confirmation of the concrete size. For example, the space height of the control platform of machine-tool is $800 \mathrm{~mm} \sim 900 \mathrm{~mm}$, and the height from the ground to the table-board of control platform is $900 \mathrm{~mm} \sim 1100 \mathrm{~mm}$, and the height from the ground to the display is $1600 \mathrm{~mm} \sim 1800 \mathrm{~mm}$, and the height of the secondary display is $200 \mathrm{~mm} \sim 300 \mathrm{~mm}$.

The working position of the digital control machine-tool is fixed in the room, and the working atmosphere is stable, so the major tonality should not be too excited and depressed, and it should adopt the warm color or cool color with low purity and middle brightness according to the environment conditions such as lighting and surrounding equipments.

To utilize the comparison partition of color area to reflect the function character of products, common metal plates should be selected to make the appearance, so the deep and "heavy" colors can be used to represent the stable and powerful functional characters.

The color of the machine should make for human various activities when human use the machine, and make operators feel good and safe, which can realize exact and reliable operation and high work efficiency, and maintain the health of body and mind.

Big pieces such as the base, the lathe bed and the pole of the machine-tool should adopt the heavy and stable color which could make human feel that the machine-tool is stable and reliable, but the components such as work platform, apron box and slipper block are always located in the place where human often work, and these components should adopt shadow color to relieve human oppression, and these components are also sports components and are dispersed in different places, and the total area is large, so they should not adopt excited and stimulated warm colors such as red, orange and yellow to avoid inducing human vision tiredness and reducing work efficiency. The color of the display apparatus in the machine should possess good visibility, and the key-press, pushbutton and various handles and hand wheels can adopt different colors for coding and distinguishing to make human quickly react and exactly operation for fulfilling the harmonized relationship of the machine.

The color design must closely combine with the manufacturing and processing method of the materials, the quality of the processed materials, and the applied of the metal pieces.

The physical characters of digital control machine-tool include clear edges and corners, and big and high bodies. To strengthen the stabilization sense and overcome the monotony sense of single color, the shell can adopt double colors, and only implement compared processing of the brightness and purity to acquire the upper bright and lower dark, and upper light and lower heavy stabilization. But the dark color proportion should be proper to avoid destroying the precision, bright and kind sense of the apparatus. The big casting products should adopt the color with middle brightness and low purity, and the painting technology is generally adopted to represent the color, which will combine with the bright and exquisite color division to acquire lively and comfortable sense. The faceplate is formed by pressing, and its surface is clean and regular, and the lines are straight, and it can adopt bright, shadow and pure color to make the 
products show efficient and extended.

For the process of small-sized exact manufacturing, the coloring technology of puttying and painting obviously can not fully reflect the performance of high-quality materials and the manufacturing technology of high level, so the various new materials (such as various engineering plastics with perfect performance) should be specially emphasized to exert the color quality, and the special processing such as various super fine machining, machinery surface processing, heat processing and chemical process about metals should be applied to reflect the level of high technology and give prominence to the modern design character of the time style.

The instruments and apparatuses have many characters such as high precision, small volume, delicate technology, good operation environment and close contact with human, so these factors should be considered comprehensively, and the color of shell and the color of faceplate should be respectively processed.

First, the shell of instrument should adopt the warm color with non-dazzle surface, high brightness and low purity, which can bring bright and kind sense to human. And the shell of the apparatuses should adopt the deep and dark color even the black color, which can bring simple and compact aesthetic feeling to human.

Second, the faceplates of instruments and apparatuses should adopt the dark color without simulation and glisten, and the components, characters, reticles and color arrangement should be clear, bright and soft. The faceplates can adopt the bright color lines with strong comparative grounding to strengthen the nicety of the visibility and the order sense of the faceplate, and enrich the color effect of faceplates. Because the black color, the white color and the color of many colors such as gold, argent, and plating chrome are easily harmonize with other colors, which can enhance the color gradation of the predominant color, strengthen the color effect and make the products more distinguished, luxury and extractive, but these color can only be used in small areas to avoid that the excessive stimulation bring adverse influences.

Some scholars found that $1 / \mathrm{f}$ turbulent fluctuation was the signal turbulent rule existing in the nature universally, and many human physiological colors all possessed $1 / \mathrm{f}$ turbulent fluctuation, and human vision and hearing all will produce good identification sense to $1 / \mathrm{f}$ turbulent fluctuation. People have found that many human physiological signals all belonged to $1 / \mathrm{f}$ noise and possessed $1 / \mathrm{f}$ turbulent fluctuation, and when human were in the loose and pleased state, the power spectra index of physiological signals $\beta$ (the negative production rate of $1 / \mathrm{f}$ ) is very close to 1 . The dulcet music possesses obvious 1/f turbulent fluctuation, which was discovered early by R.F.vossd in 1975, and in 1998, Jeong JS and Joung MK studied how human music sensibility was reflected in the electric signals of brain, and the research results again proved that the music with $1 / \mathrm{f}$ turbulent fluctuation could bring human pleased response.

\section{Design of the digital control machine-tool operating system}

The system comprehensive evaluation of the design scheme should be made from the functions (production, safety and usage) and the tastes (modeling, color and psychological reception).

For the appearance modeling of the digital control machine-tool, the total modeling should be concise and stable, and local parts use the modeling languages such as line accession and incision to bring bright and sports sense, and the various function sections can be distinguished by modeling characters and colors to bring better definition and security for users.

The operating interface adopts the folio face design, which can not only integrate with the sliding door, but more accord with the requirement of ergonomics. The single-side sliding door design is adopted, and the chips escape pipes are distinguished from the color and principle structure. The mobile parts all are concealed to strengthen the integration and security of the conformation. The continual arc lines are added to break traditional beeline design, weaken the fixed inhospitality of the machine and strengthen the appetency between human and machine, and the power of the arc lines are straight to make the machine possess the basic symbols of operation. The color of the machine body adopts the bright and neutral cool grey color, and the brightness of the bottom base can be reduced to avoid the error operations because of glare, glisten or vision tiredness, and complement the stability. The view window is in the middle, and the interlayer can be introduced when pushing forward the left and the design will not influence the concise appearance under dynamic state and static state. To keep the integration, the control platform adopts the hidden hanging form to make the wiring in the right interlayer. There are respective an inlet orifice at the left and the right, and in the normal state, they are riveted by the bolts which are removed when the machine is used. The motor is moved down to weaken its huge sense, and the wedges machine cover is installed on the top to maintain the integrated image.

For the color design, the navy blue and carmine are used to make the body of machine still and steady, and make operators feel calm and rational. The yellow alert belt indicates the work area, and the bold and forceful colors can be attempted to change the depressed and bald distribution of the workshop and imply the importance of digital control machine-tool. The sheet metal parts decoration on the surface could enrich the surface effect and strengthen the comparison of materials and mechanism in the workshop and the horizontal line can stabilize the vision and reduce the height of vision. And the bright color of the window can bring out the best in each other. The color of the chips escape pipe is deeper than the color of the body, which can indicate the function distinguish and add the stabilization of vision. 
The sound design adopts the supply air bottom equipment which could generate four wind speed changes. The human heat comfort experiment results indicated that the wind speed change mode close to the natural wind spectra character had better acceptability than other three wind change modes (stable wind speed, sine wind speed and random wind speed), and $61 \%$ testers thought the natural wind was the most comfortable. In the frequency analysis of the simulated natural wind, the power spectra density curve basically fulfills the $1 / \mathrm{f}$ rule.

Through comprehensively and systematically analyzing of the digital control machine-tool operating system, combining with the design concepts of the system theory and the methodology, the design is human oriented and the flexible layout is adopted to realize the green design and make the operation more convenient and comfort. According to human physiological and physical characters, the design in the article more accords with human psychology, which can enhance human work efficiency and the economic benefits of enterprise and make the machine-tool system to operate in the better state.

\section{References}

Chen, Hanqing. (1995). Vision Communication Design. Beijing: Popular Science Press.

Jian Zhaoquan. (2000). Industrial Design Methodology. Beijing: Beijing Institute of Technology Press.

Liu, Guanzhong. (1994). An Introduction to Industrial Design. Beijing: China Science \& Technology Press.

Xiao, Yunlong. (1989). Creative Design. Wuhan: Hubei Science and Technology Press.

Yin, Dingbang. (2000). An Introduction to Design Science. Changsha: Hunan Science and Technology Press.

Zhao, Hongiiang. (2005). Meanings of Design Art. Changsha: Hunan University Press. 